\title{
De la grande ville à la grande politique : La dimension constructive du conflit dans les écrits de Georg Simmel et de Karl Mannheim
}

Par GABOR TVERDOTA

Université catholique de Louvain

\section{Introduction}

Dans ce travail, je me propose d'étudier un problème qui ne fait pas partie des thématiques de prédilection des penseurs que l'on classe parmi les critiques de la modernité. Pourtant, au vu de son importance dans la période historique qui a vu naître la Kultrukritik, il peut paraître surprenant qu'aussi peu d'attention lui ait été accordée. Le phénomène en question est celui du conflit, que j'aborderai au prisme de deux lectures qui en ont été faites. La première, plus générale, et qui a sans doute eu une influence plus considérable, est celle de Georg Simmel. La deuxième, centrée sur un problème plus particulier, sans être pour autant moins intéressante, peut être comprise comme une application des thèses de la première. Cette seconde lecture est proposée par Karl Mannheim, cofondateur, avec Max Scheler, du courant de la sociologie de la connaissance.

Ces deux tentatives de théorisation du conflit ont chacune une grande cohérence interne, mais peuvent paraître très problématiques si on les considère rétrospectivement, au vu des événements qui ont façonné le $\mathrm{XX}^{\mathrm{e}}$ siècle, particulièrement la Deuxième Guerre Mondiale et la Shoah. De ce point de vue, il est significatif que la période envisagée dans ce séminaire s'étende jusqu'en 1930. Si l'on y ajoute trois années, il s'agirait sans doute de la date limite jusqu'à laquelle l'on pouvait encore escompter ce qu'au-delà de ce seuil symbolique seuls les anges et les ignares pouvaient espérer. Ceci 
est vrai dans une plus grande mesure, nous le verrons, pour la tentative mannheimienne de la théorisation du conflit.

Dans mon étude, j'essayerai principalement de montrer deux choses. La première concerne la logique interne de ces deux lectures, ainsi que les présupposés anthropologiques qui leur sont communs. La seconde a trait à ce que je tenterai de décrire comme leur point aveugle. L'objectif de ce travail est de synthétiser ces deux aspects de sorte à parvenir à une décantation de ce que l'on peut retenir d'utile des deux théories étudiées.

\section{La grande ville}

Dès le début de sa conférence sur la grande ville et la vie de l'esprit, Simmel signale la portée quasi-tragique de la question discutée. La première phrase du texte annonce :

Les problèmes les plus profonds de la vie moderne prennent leur source dans la prétention de l'individu à affirmer l'autonomie et la spécificité de son existence face aux excès de pouvoir de la société, de l'héritage historique, de la culture et de la technique venue de l'extérieur de la vie - figure ultime du combat contre la nature que l'homme primitif doit mener pour son existence physique ${ }^{1}$.

Outre le ton grave et tragique, il convient de relever que l'incipit de l'étude définit d'emblée et très clairement la perspective dans laquelle il convient de traiter le phénomène de la grande ville moderne. Il s'agit de l'anthropologie à laquelle - dans la mesure où elle vise à dégager le noyau apriorique des opérations qui rendent possible l'existence de l'homme et de la société - il n'est pas tout à fait déplacé d'ajouter l'épithète de "phénoménologique 》. Concrètement, cela exige du chercheur d'interpréter chaque élément de la crise dans laquelle l'homme moderne de la grande métropole se trouve à la lumière d'une situation de crise archaïque construite sur le modèle des expériences de pensée des théories contractualistes classiques, et dont, en un sens bien précis ${ }^{2}$, il procède. Au niveau de l'exposition — et de la rhétorique

${ }^{1}$ Georg Simmel, Les Grandes villes et la vie de l'esprit, trad. fr. J.-L. VieillardBaron, Paris, Éditions Payot \& Rivages, 2013, p. 39 (nous soulignons).

${ }^{2}$ Le but de ce type de construction n'est nullement de retracer la genèse factuelle d'une situation donnée à partir de la connaissance historique d'une situation précédente, mais assure une double tâche. 1) La première tâche est d'ordre pédagogique : c'est en effet ce type de narration qui assure le mieux l'intelligibilité de la situation 
qui y correspond -, cette démarche doit suggérer qu'entre le commencement du processus de l'hominisation et l'état actuel de la culture, la différence n'est au fond que quantitative.

Quel est l'élément formel qui permet ce rapprochement fulgurant? Cet élément commun, je l'appellerai, à la suite de Hans Blumenberg — lecteur assidu de Simmel - la " situation rhétorique »" La situation rhétorique est le principe de réalité pour l'être indigent qu'est l'homme. L'essence de ce principe peut être exprimée dans la formule suivante : il ne va pas de soi que l'homme puisse exister ${ }^{2}$ ou, dit avec les mots de Simmel :

[L]'insertion de l'humain dans les données naturelles du monde, au contraire de l'animal, ne s'opère pas sans problèmes : il s'en arrache, s'y oppose, il exige, il lutte, il exerce et subit la violence : ce premier grand dualisme est au commencement du processus qui se déroule indéfiniment entre le sujet et l'objet $^{3}$.

De cet état de fait, il découle que ce qui définit formellement la situation rhétorique, c'est la présence conjuguée de la contrainte d'agir et du défaut de normes claires de l'action. Dès lors, les fonctions élémentaires de toute rhétorique sont au nombre de deux : 1) tout d'abord, faire gagner du temps et de l'espace de jeu pour préparer les actions nécessaires à la survie;

que l'on veut appréhender. 2) La deuxième tâche est d'ordre matériel : ce procédé désire en effet isoler les constituants de base de la situation et déterminer leurs interconnexions du point de vue de leur signification. La dérivation de la deuxième situation (l'homme dans la grande ville) à partir de la première (l'homme archaïque) ne se réfère qu'en apparence au temps historique, et sert en réalité à établir une généalogie, c'est-à-dire à offrir une description de la logique interne des interconnexions qui constituent le phénomène à décrire. Sur ce point, voir Karl Mannheim, Structures of Thinking, trad. angl. Jeremy J. Shapiro et Shierry Weber Nicholsen, édité et introduit par David Kettler, Volker Meja et Nico Stehr, Londres/Boston/ Henley, Routledge and Kegan Paul, 1982, p. 205.

${ }^{1}$ Hans Blumenberg, "Actualité d'une approche anthropologique de la rhétorique », dans Id., L'Imitation de la nature et autres essais esthétiques, trad. fr. I Kalinowski et M. B. de Launay, Paris, Éditions Hermann, 2010, p. 103.

${ }^{2}$ Ibid., p. 103-104.

${ }^{3}$ Georg Simmel, « Le Concept et la tragédie de la culture », dans Id., La Tragédie de la culture, trad. fr. S. Cornille et P. Ivernel, Paris, Éditions Payot \& Rivages, 1988, p. 179. 
2) apprendre à maîtriser les situations aiguës avant qu'elles n'interviennent, précisément - et dans l'idéal - pour qu'elles n'interviennent pas ${ }^{1}$.

D'un point de vue matériel, le point commun entre la situation anthropologique originaire et celle de l'individu dans la grande ville est sans doute l'excès de sollicitations auquel ce dernier doit fournir une réponse adaptée. Les phénomènes d'accélération tant décriés de la vie moderne détiennent la même fonction que le débordement de stimulations auquel l'être biologiquement indigent qu'est l'homme a dû faire face au moment de la rupture avec le monde animal. Ainsi, Simmel désigne "l'intensification de la vie nerveuse, qui résulte du changement rapide et ininterrompu des impressions externes et internes $»^{2}$ comme le phénomène central de la condition métropolitaine. Cette intensification plonge l'homme dans un cruel embarras qui le force à aiguiser son intellect, « [cet] organe de protection contre le déracinement dont le menacent les courants et les discordances de son milieu extérieur $»^{3}$, au détriment de sa sensibilité.

Il y aurait beaucoup à dire sur les belles descriptions concernant les conséquences de l'adaptation par le biais de l'intellectualisation de la vie de l'âme, mais je me concentrerai ici exclusivement sur les incidences $p s y$ chiques et intersubjectives de ce phénomène. À ce niveau, l'autoconservation du sujet face à la grande ville réclame de lui un comportement de nature à première vue tout à fait asociale. Simmel désigne ce comportement en tant que caractère réservé et explique sa nécessité de la façon suivante :

Si la rencontre extérieure et continuelle d'un nombre incalculable d'êtres humains devait entrainer autant de réactions intérieures que dans la petite ville, où l'on connaît presque chaque personne rencontrée et où l'on a un rapport positif à chacun, on s'atomiserait complètement intérieurement et on tomberait dans une constitution de l'âme tout à fait inimaginable ${ }^{4}$.

Le principe d'économie contraint donc l'individu de la grande ville à se préserver d'une trop grande ouverture à l'égard d'autrui. Mais le foisonnement de rencontres intersubjectives n'est pas l'unique cause de la réserve. L'indétermination du comportement d'autrui en est une seconde, non moins

\footnotetext{
${ }^{1}$ Hans Blumenberg, Description de l'homme, trad. fr. D. Trierweiler, Paris, Éditions du Cerf, 2011, p. 552.

${ }^{2}$ Georg Simmel, Les Grandes villes et la vie de l'esprit, op. cit., p. 41 (souligné dans le texte).

${ }^{3}$ Idem.

${ }^{4}$ Ibid., p. 53.
} 
importante ${ }^{1}$. Étant donné que l'homme est incapable de trancher avec assurance la question de savoir si l'autre en face de lui sera un ami ou un ennemi, il est préférable pour lui d'adopter une posture qui permette de maintenir le plus longtemps possible cette indétermination, qui préserve précisément du pire, à savoir de l'affrontement physique que pourrait amener une mauvaise évaluation des intentions d'autrui, ou un mauvais geste de la part de celui qui s'élance vers l'autre. L'antipathie prononcée que ressentent bien souvent les habitants de la grande ville les uns envers les autres n'est que le versant subjectif de la réserve dont la fonction essentielle est de minimiser la pression d'agir qui s'exerce sur l'homme dans une situation d'indétermination. En effet, sans cette légère aversion, cette hostilité ou cette répulsion réciproques, la multiplication des rencontres de proximité avec autrui,

tournerait aussitôt en haine et en combat. [...] [N]ous sommes préservés de [ce] danger lié à la grande ville par l'antipathie, antagonisme latent et phase préliminaire de l'antagonisme de fait; elle produit les distances et les éloignements sans lesquels nous ne pourrions tout bonnement pas mener ce genre de vie ${ }^{2}$.

Ce passage contient en vérité le principe même de la sociologie du conflit de Simmel. Le sociologue montre en effet que ce qui apparait extérieurement sous la forme de la réserve et qui est vécue - souvent de manière inconsciente - comme de l'antipathie, a pour fonction essentielle de produire les éloignements, les distanciations et le gain de temps nécessaires au maintien de l'indétermination des rapports ami-ennemi, préservant de la haine et des réactions de prévention qu'une trop grande proximité susciteraient chez le citadin.

De ce raisonnement, nous ne retiendrons que la conclusion suivante : il existe, d'après Simmel, des formes de conflits dans la société dont la fonction est l'évitement d'un autre conflit, qui risquerait de s'avérer immaîtrisable, s'il éclatait. C'est pourquoi, « ce qui apparaît immédiatement en celle-ci [= l'antipathie] comme dissociation n'est ainsi en réalité qu'une de ses formes élémentaires de socialisation $»^{3}$.

${ }^{1}$ Sur la catégorie anthropologique de l'indétermination, voir Hans Blumenberg, Description de l'homme, op. cit., p. 243-244, 291, 784.

${ }^{2}$ Georg Simmel, Les Grandes villes et la vie de l'esprit, op. cit., p. 55.

${ }^{3}$ Idem. 


\section{Le conflit}

Dans son ouvrage intitulé Le Conflit, Simmel généralise les acquis de son étude sur la grande ville. En usant d'une métaphore fondamentale, il assimile la société à un organisme et le conflit à la maladie : tout comme l'organisme fait sa maladie pour guérir, la société se donne ses conflits pour pouvoir exister. Pour Simmel, le conflit vise le rétablissement de l'état d'équilibre de la société, il est déjà

la résolution des tensions entre les contraires; le fait qu'il vise la paix n'est qu'une expression parmi d'autres, particulièrement évidente, du fait qu'il est une synthèse d'éléments, un contre autrui qu'il faut ranger avec un pour autrui sous un seul concept supérieur ${ }^{1}$.

En d'autres termes, le conflit, en tant que divergence, fait inextricablement partie d'une totalité qui recèle en elle un élément de convergence. De ce fait, le conflit est un facteur d'équilibre sans lequel la société ne serait même pas envisageable.

Le phénomène qui manifeste de la manière la plus transparente le caractère socialisant du conflit est la concurrence. Si l'on voulait donner une définition formelle de la concurrence, on dirait qu'elle est une lutte indirecte qui consiste « dans les efforts parallèles des deux parties en vue d'un seul et même enjeu $»^{2}$. La concurrence réalise ce miracle de la culture humaine qui consiste en ceci que l'opposition, fût-elle la plus totale, entre deux parties en lutte résulte en un gain pour une instance tierce. En d'autres termes, à côté de la simple victoire sur l'autre, l'apparition d'une valeur objective indépendante en elle-même du combat est la condition sine qua non pour qualifier une lutte de concurrentielle. Simmel le montre négativement à l'aide des deux exemples suivants : le commerçant qui discrédite son concurrent en faisant courir le bruit qu'il est insolvable n'a absolument rien gagné si sa marchandise est à tel point piètre que les consommateurs s'en détournent. Pour qu'il vende ses produits, il doit aussi bien contenter sa clientèle. L'amant qui ridiculise son rival n'a encore rien gagné si la dame lui refuse tout simplement ses faveurs. Il doit se montrer non seulement meilleur que son rival, mais également digne de l'amour de la dame de son cœur.

Selon Simmel, dans le monde moderne, les effets socialisants de la concurrence sont d'autant plus indispensables que la taille et le degré

${ }^{1}$ Georg Simmel, Le Conflit, trad. fr. S. Muller, Paris, Éditions Circé, 2003, p. 20.

${ }^{2}$ Ibid., p. 72. 
d'individualisation des sociétés (nous pouvons repenser ici à la situation de l'individu de la grande ville) font que

bon nombre d'intérêts, qui maintiennent finalement la cohésion du cercle de chaînon en chaînon, ne sont vivants que si la lutte concurrentielle est assez désespérée et assez violente pour les imposer au sujet. [...] [I]l semble que les efforts des hommes pour les autres, l'adaptation des uns aux autres, ne sont possibles en effet qu'au prix de la concurrence ${ }^{1}$.

Là encore, c'est la même idée qui revient : l'intensification de la concurrence - la production d'un bien pour la société à partir d'une simple situation d'opposition entre deux individus — dans le monde moderne apparaît comme le moyen le plus adéquat pour éviter que faute d'une puissance de cohésion positive, les forces vraiment «désocialisantes» ne prennent le dessus.

\section{La sociologie de la connaissance}

L'idée simmelienne selon laquelle l'intérêt que portent les hommes les uns aux autres, c'est-à-dire la possibilité même de forger un monde commun, repose sur l'existence de la concurrence, constitue l'un des postulats de base de la sociologie de la connaissance. Toutefois, la façon dont Mannheim va infléchir cette constatation est assez originale : à savoir, en opérant un détour par Marx, ce dernier introduisant l'idée bien connue que c'est l'être social qui détermine la conscience des hommes et non l'inverse, et inventant du même coup l'instrument de lutte connu sous le nom de la critique des idéologies. Or, selon Mannheim, si l'on généralise la critique des idéologies, cela mène nécessairement à la découverte que toute pensée, la nôtre y comprise, est idéologique, au sens où elle est liée aux aspirations et volitions de base qui découlent en grande partie de la situation sociale de chaque acteur politique. La sociologie de la connaissance prend naissance dans cet acte de généralisation de la critique des idéologies, dont résultent le relativisme et le nihilisme, mais elle dépasse la simple doctrine des idéologies en comprenant que la partialité ou la fausseté des énoncés de l'adversaire - ou des siens propres - ne découlent pas toujours d'une dissimulation intentionnelle, mais des différences qui existent inéluctablement entre la structure de la conscience des sujets occupant des places fort diverses dans le processus socio-historique. Selon Mannheim, l'une des raisons de la

${ }^{1}$ Ibid., p. 78. 
crise scientifique et politique de l'époque moderne - dont témoignent entre autres l'acharnement des luttes idéologiques ainsi que l'apparition du relativisme dans la science et la philosophie - est l'écart parfois presque insurmontable qui s'est creusé entre les visions-du-monde en conflit. Le but de la sociologie de la connaissance est précisément de trouver une médiation entre ces dernières par le biais du rétablissement de l'objectivité perdue : ainsi, ce que deux sujets socio-historiques donnent à voir de manière correcte mais différente en raison des divergences de la structuration interne de leur vision, il convient de le comprendre précisément en fonction de cette structuration en convertissant - en traduisant - l'une dans l'autre.

Or, que cette traduction soit possible, c'est précisément l'existence de la concurrence qui le garantit. Pourquoi ? La réponse est que, la concurrence se définissant comme les efforts parallèles d'au moins deux parties en vue d'un seul et même enjeu, cela - à savoir l'enjeu et ce qu'il faut mettre en œuvre pour la victoire - fournit un élément commun qui assure la traductibilité des perspectives. Quel est cet enjeu d'après Mannheim ? Dans sa conférence prononcée en 1928 et intitulée «De la concurrence et de sa signification dans le domaine de l'esprit », le sociologue écrit que cet enjeu est « la possession du juste point de vue (social), ou tout au moins le prestige de cet apanage $»^{1}$, ce qu'un peu plus loin le sociologue reformule, pour des raisons polémiques évidentes, en empruntant le terme d'Öffentlichkeit à Heidegger, de la manière suivante : «Les partenaires et concurrents dans ce domaine luttent constamment pour "l'interprétation de l'être dans l'espace public" $»^{2}$. Et Mannheim de commenter ainsi :

D'un point de vue philosophique, il se peut qu'il en aille autrement, mais du point de vue de la science de la société, tout savoir historique, toute vision-dumonde, tout savoir sociologique — seraient-ils l'exactitude et la vérité absolues mêmes - sont enchâssés dans et portés par l'instinct de pouvoir et de valorisation de certains groupes concrets qui veulent faire de leur interprétation du monde celle de l'espace public. [...] [M]ême l'interprétation « juste», « scientifique » de l'être ne naît pas par pure soif de savoir dans la

${ }^{1}$ Karl Mannheim, «De la concurrence et de sa signification dans le domaine de l'esprit », trad. fr. J.-L. Évard, dans L'Homme et la société, n 140-141, 2001/2-3, p. 62.

${ }^{2}$ Idem. 
contemplation, elle remplit bien plutôt une fonction originaire, sans âge : permettre de s'orienter dans le monde ${ }^{1}$.

Le conflit des interprétations de l'être sous la forme de la concurrence dans le domaine des idées désigne ainsi pour Mannheim de manière inséparable à la fois les divergences qui se manifestent dans les sociétés sur le sens de la communauté du monde et un horizon d'entente qui transparaît là-même où le conflit se fait le plus aigu ${ }^{2}$. Pour le dire plus simplement, un horizon commun est possible précisément parce que des divergences existent quant à son sens. Dans la même conférence, Mannheim dresse un tableau de l'évolution historique des formes dominantes de la concurrence pour l'interprétation de l'être dans l'espace public, suggérant que les tendances dissociantes et synthétisantes, bien que constitutives du phénomène de la concurrence, ne sont pas présentes de manière égale dans les diverses phases de l'histoire occidentale. Le relativisme et le nihilisme, ainsi que le sentiment de désespoir qui les accompagne, sont précisément les signes d'une atomisation extrême de l'espace public contemporain. Cependant, suggère Mannheim, la diffusion croissante des idées fondamentales de la sociologie de la connaissance - surtout de l'idée de la pensée solidaire-de-l'être-social - est la meilleure preuve de la présence égale de tendances à la synthèse qui vont à l'encontre des phénomènes si difficilement supportables du dialogue de sourds entre les visions-du-monde en conflit et de la critique sans merci des idéologies.

En somme, dans sa conférence de 1928 sur la concurrence dans le domaine de l'esprit, Mannheim propose une sorte de pacte aux membres présents et absents de l'intelligentsia allemande : en échange du renoncement à la pureté du savoir, qui équivaut à l'acceptation de son rattachement à son lieu constitutif dans le social - en d'autres termes, à l'acceptation du caractère constitutif de la conflictualité dans les productions de l'esprit Mannheim promet aux intellectuels une sortie vers l'avant des apories du relativisme. En effet, dès lors que les intellectuels s'accorderaient sur la convergence de cet intérêt primaire (la sortie du relativisme), conformément à la loi de la concurrence à la performance, ils ne pourraient pas ne pas

\footnotetext{
${ }^{1}$ Ibid., p. 63 (souligné dans le texte). L'idée que Mannheim mobilise ici est d'origine nietzschéenne. Voir Friedrich Nietzsche, La Volonté de puissance, tome I, texte établi par Friedrich Würzbach, trad. fr. G. Bianquis, Paris, Editions Gallimard, 1995, Livre II, "Morphologie et évolution de la volonté de puissance », aphorismes $\mathrm{n}^{\circ} 130-134$, p. $264-265$.

2 Jean-Luc Évard, "L'Éclipse double», dans L'Homme et la société, n ${ }^{\circ} 140-141$, 2001/2-3, p. 30-31 et 41 .
} 
adopter la perspective de la sociologie de la connaissance comme meilleur moyen de dépasser l'état de la concurrence atomisée qui mène, justement, au relativisme. L'aveu ainsi arraché aux intellectuels des effets de la concurrence dans leur propre domaine ne pourrait pas non plus manquer de leur faire prendre conscience du fait que, du coup, le destin de la culture et le progrès de la connaissance sont liés à leur propre conscience sociale et politique. Cette prise de conscience devrait également donner les outils à même de transformer le champ des idées politiques. Il s'agit de trouver une base de médiation qui permette d'éviter les phénomènes de violence symptomatiques de la situation de crise qui découle du discrédit mutuel des idéologies ${ }^{1}$.

Le projet de Mannheim escomptait en somme que la création d'une plateforme d'entente commune minimale irriguerait les tendances analogues à la synthèse dans la grande politique. Cette supposition audacieuse, sinon naïve, se fonde sur le présupposé fort problématique d'une convergence entre la volonté scientifique et la volonté politique, convergence pour laquelle la médiation assurée par la sociologie de la connaissance est certes nécessaire, mais qui suppose néanmoins que l'horizon commun des deux volontés ne peut être autre que la régulation rationnelle de l'agonistique des intérêts. Au sein de ce mouvement général de "rationalisation» de l'agir, la sociologie de la connaissance aurait eu pour but pratique d'ouvrir la voie à une connaissance anticipée du conflit ${ }^{2}$. Son action spécifique aurait consisté à déblayer le terrain pour les décisions politiques, qui tendent par nature à prendre le chemin le plus court et à user d'une violence directe là-même où cela pourrait être évité. En quoi consiste plus précisément ce savoir politique ? La réponse de Mannheim est la suivante :

La qualité la plus spécifique de la connaissance politique réside en ceci que l'expansion du savoir ne rend pas superflue la décision, mais ne fait que la repousser de plus en plus, et ce que nous gagnons dans ce repli demeure sous la forme de l'élargissement de l'horizon et d'un savoir acquis ; des avancées de l'exploration sociologique des idéologies, nous sommes en droit d'espérer que les connexions jusqu'à présent peu étudiées de la position sociale, des impulsions, de la volonté et de la perspective deviendront de plus en plus transparentes; que [...] la volonté collective et la pensée qui y correspond sera mesurable avec une très grande précision, et enfin que les modes de réaction idéologiques des couches sociales seront largement prévisibles. Ces fondements de la sociologie de la connaissance n'annulent pas, tant s'en faut,

${ }^{1}$ Ibid., p. 33.

${ }^{2}$ Ibid., p 38. 
notre propre décision ; simplement, le champ de vision dans lequel on a à la prendre $s$ 'élargit considérablement ${ }^{1}$.

En considérant les effets visés par la politique scientifique préconisée par Mannheim - tels que : le gain de temps par le biais du repoussement du moment de la décision, la prise de distance par rapport à ce qui est potentiellement menaçant, la transparence des divers facteurs d'une constellation problématique et enfin la prévisibilité des réactions des acteurs -, on reconnaîtra aisément les éléments déterminants relevés par Simmel dans ce que nous avons appelé la situation rhétorique de l'individu dans la grande ville. Des tournures apparaissant dans d'autres textes, décrivant la sociologie de la connaissance comme «l'instrument d'élargissement de la conscience et de l'âme » ou comme «l'organon de la nouvelle hominisation », renforcent encore l'intuition que les théories mannheimienne et simmelienne du conflit proviennent en effet de la même matrice anthropologique.

Il est patent que malgré leurs présupposés anthropologiques pessimistes sur l'homme en tant qu'être indigent, tant la théorie du conflit de Simmel que son application dans le domaine de la sociologie politique par Mannheim sont foncièrement optimistes du point de vue de la possibilité du dépassement de la crise permanente que constitue le processus de l'hominisation. Au vu de l'échec pratique de la sociologie de la connaissance de Mannheim, il est d'autant plus important aujourd'hui de reconsidérer l'ensemble des postulats sur lesquels elle se fondait. En effet, il semblerait que l'échec de ce projet, qui se concevait pourtant comme la doctrine la plus appropriée du monde moderne, ne soit pas simplement un hasard. Au-delà de son anéantissement brutal par le biais de la persécution physique de ses représentants, souvent d'origine juive, il me semble que son échec est dû bien plutôt au succès fulgurant de son adversaire, et précisément sur le plan où la Wissenssoziologie désirait agir. Le national-socialisme a en effet réussi ce qui n'était qu'un vœux pieux pour Mannheim : dépasser définitivement l'impasse idéologique de la République de Weimar; quant à la fonction synthétisante et de rationalisation de la science politique, elle a été tournée en dérision par l'adhésion massive à la Weltanschauung de l'hitlérisme, ce dernier capable de mobiliser des millions de personnes de toutes les classes sociales émotionnellement disposées à la mythisation des idéologies ${ }^{2}$.

${ }^{1}$ Karl Mannheim, Idéologie et utopie, trad. fr. J.-L. Évard, Paris, Éditions de la Maison des Sciences de l'Homme, 2006, p. 156 (nous soulignons).

${ }^{2}$ David Kettler, Volker Meja, Nico Stehr, Karl Mannheim, trad. fr. E. Treves, Paris, PUF, 1987, p. 90. 


\section{Le point aveugle des théories du conflit de Simmel et de Mannheim}

Pour comprendre la raison de la cécité de Mannheim non pas tant devant le danger représenté par le national-socialisme (il en était parfaitement conscient), mais plutôt devant l'imminence de sa victoire, il me semble fructueux de questionner ce que j'ai appelé dans l'introduction de cette étude le «point aveugle» de la théorie du conflit de Simmel. Qu'est-ce donc que cette théorie ne voit pas? Pour le montrer, je renverrai à une distinction cruciale faite par Simmel dans son essai sur le conflit entre le combat motivé par un terminus ad quem objectif et celui motivé par un terminus a quo subjectif ${ }^{1}$.

Des deux types de conflit, seul le premier est en adéquation avec la nature sociable de l'homme. Sa caractéristique principale est en effet d'être non seulement ouvert à des normes communes et à des restrictions, mais surtout de pouvoir être substitué par d'autres moyens. Cela exprimé avec les mots de Simmel :

Quand le but en question est extérieur à lui-même, le fait que toute fin peut être atteinte en principe par des moyens différents donne une certaine coloration au combat. [...] Lorsque le combat n'est qu'un moyen, déterminé par son terminus ad quem, il n'y a pas de raison de ne pas le limiter ou de ne pas s'en abstenir, puisqu'il peut être remplacé par un autre moyen avec le même succès ${ }^{2}$.

La possibilité de la substitution métaphorico-symbolique du conflit ouvre la voie à l'évitement de ce que Simmel appelle la «conséquence la plus impitoyable du rapport de forces $»^{3}$, c'est-à-dire la mort de l'autre. Cette possibilité constitue simultanément la condition logique de toute situation rhétorique, y compris celle de la sociologie de la connaissance, dans la mesure où elle rend possible la minimisation de la pression d'agir par le biais de la substitution de performances verbales à des actions physiques ${ }^{4}$. Ce type de lutte satisfait également aux exigences de la métaphore organique de Simmel, puisque le cycle du conflit se termine par le rétablissement de l'homéostasie, pour le maintien de laquelle précisément le conflit avait éclaté :

\footnotetext{
${ }^{1}$ Georg Simmel, Le Conflit, op. cit., p. 37.

${ }^{2}$ Ibid., p. 37.

${ }^{3}$ Ibid., p. 145.

${ }^{4}$ Hans Blumenberg, «Actualité d'une approche anthropologique de la rhétorique », art. cit., p. 103.
} 
Une fois le conflit terminé de l'une des manières habituelles — victoire et défaite, réconciliation, compromis - , cette structure psychique se recompose en structure de paix, le point central communique aux énergies impliquées son passage de l'excitation à l'apaisement ${ }^{1}$.

Il en va très différemment avec le second type de combat, motivé par un terminus a quo subjectif. En effet,

lorsqu[e le combat] est déterminé exclusivement pas son terminus a quo subjectif, lorsqu'il y a des énergies internes qui ne peuvent être satisfaites que par le combat en tant que tel - alors il est impossible de lui substituer autre chose, il est sa propre fin, son propre contenu, et il ne vient donc s'y ajouter aucune autre forme. Ce type de combat pour l'amour du combat semble être entraîné par une certaine pulsion formelle d'hostilité qui s'impose parfois à l'observation psychologique ${ }^{2}$.

Ce qui caractérise ce deuxième type de conflit décrit par Simmel c'est que, contrairement au premier, il ne peut être intégré au cycle de l'homéostasie sociale dans la mesure où l'on ne trouve en lui que l'élément du contre autrui, sans celui du pour autrui, c'est-à-dire qu'il est impossible de le subsumer sous un seul concept supérieur, celui de la société. Pour le dire plus simplement, à ce genre de combat, l'élément socialisant fait complètement défaut.

La façon dont Simmel essaye de composer avec ce fait dérangeant qui contredit les postulats de sa théorie du conflit est significative car foncièrement défensive. Le premier pas de sa stratégie consiste à dire que ce genre de pulsion à l'hostilité, à la haine pure de l'autre, n'est qu'un résidu du processus de l'hominisation, au cours duquel nous avons si souvent été appelé au combat que cette poussée à la haine immotivée de l'autre s'est décantée dans le fond héréditaire de notre espèce. Il s'agit de surcroît d'un résidu qui ne s'éveille qu'occasionnellement chez certains individus qui, de toute façon, verseront leurs énergies dans la lutte du premier type dès qu'un bien extérieur éveille leur convoitise ${ }^{3}$. Plus significatif est toutefois que Simmel veille à désamorcer par là une autre hypothèse, à savoir celle d'une

${ }^{1}$ Georg Simmel, Le Conflit, op. cit., p. 139.

${ }^{2}$ Ibid., p. 37-38.

${ }^{3}$ Ibid., p. 46. 
socialité fondée sur le conflit pour le conflit, sur ladite "haine pure de l'autre $»^{1}$. Ainsi, écrit-il,

[j]e ne connais en fait qu'un seul cas où le plaisir du combat et de la victoire en tant que tel est un motif exclusif, alors que d'ordinaire il n'est qu'un élément dans les antagonismes causés par un contenu : le combat ludique, et plus exactement celui où le vainqueur ne remporte aucun prix extérieur au jeu lui-même ${ }^{2}$.

Ainsi, l'unique exception imaginable où le conflit pour le conflit est facteur de socialisation, c'est le cas totalement anodin du combat ludique.

Je crois bien que c'est ici que réside le point aveugle tant de la théorie du conflit de Simmel que de la théorie de la concurrence de Mannheim. En effet, elles sont toutes les deux réticentes à prendre en considération l'existence d'un type humain dont les comportements ne sont pas interprétables avec les instruments fournis par l'anthropologie de l'être indigent. La possibilité, réalisée par le national-socialisme, d'une sociabilité fondée sur le seul pouvoir rassemblant de la haine de l'autre, le concept d'une unité adialectique entendue comme incorporation collective, va à l'encontre des prémisses mêmes de cette anthropologie. Bien entendu, si l'on prend le sujet rationnel fictif de l'étude de Simmel sur la grande ville comme le prototype même de l'être humain, «la conséquence la plus impitoyable» de la rencontre intraspécifique semble être en effet évitable et des enseignements permettant de répondre à la question qui sous-tend le texte, à savoir : "Comment se tirer d'affaire dans de telles conditions ? " peuvent en effet se transmettre. De même, si l'on prend le type de «l'intellectuel relativement détaché » de Mannheim comme prototype du sujet politique actif, c'est en effet la sociologie de la connaissance qui offre la modalité la plus efficace du règlement des conflits d'intérêts, ainsi que la meilleure orientation pédagogique et politique pour la formation d'une communauté humaine. Seulement, il suffit de considérer la normalisation contemporaine des formes de la socialisation basées sur des délires collectifs destructeurs, ainsi que l'impudence démesurée et croissante des puissants pour ressentir à quel point les stratégies de règlement des situations conflictuelles proposées par Simmel et Mannheim peuvent s'avérer insuffisantes devant la réalité politique actuelle.

\footnotetext{
${ }^{1}$ Sur la question d'une sociabilité fondée sur le pouvoir rassemblant de la haine nue, voir les analyses que Jacques Rancière consacre au phénomène de l'okhlos dans Aux bords du politique, Paris, Éditions La Fabrique, 1998.

${ }^{2}$ Georg Simmel, Le Conflit, op. cit., p. 46 (nous soulignons).
} 


\section{Conclusion}

Malgré cette critique que l'on peut adresser à ces deux théories du conflit social, il convient de souligner également leur pertinence dans les domaines plus restreints auxquels elles se rapportent de prime abord. Les descriptions par Simmel de la vie dans la grande ville sont d'une précision et d'une richesse incontestables, tandis que la modélisation des formes de conflit interne entre " les pôles actifs de l'interprétation de l'être » - en d'autres termes, au sein de l'intelligentsia - peut être considérée comme révolutionnaire. (En fait foi, indirectement, la résistance qu'elle rencontre encore de nos jours). Ainsi, la participation active des intellectuels dans les conflits sociaux, l'éveil de leur conscience quant aux déterminations inconscientes de leurs actions et des interdépendances secrètes qui les unissent, ou encore les méthodes de formation des acteurs collectifs, sont autant de questions qui ne cessent de se poser à nous aujourd'hui, et pour lesquelles de précieux éléments de réponse peuvent être trouvés dans les travaux de Georg Simmel et de Karl Mannheim. 